Cahiers $d u$ MONDE RUSSE

\section{Cahiers du monde russe}

Russie - Empire russe - Union soviétique et États indépendants

59/2-3 | 2018

Hors plan : L'économie informelle en URSS

\title{
Rupture du monopole dans la fabrication du cinéma (1958-1970)
}

Studios institutionnels et pratiques économiques alternatives

Making movies outside state monopoly: Institutional film studios and alternative economic practices (1958-1970)

\section{Irina Tcherneva}

\section{OpenEdition \\ Journals}

\section{Édition électronique}

URL : https://journals.openedition.org/monderusse/10434

DOI : $10.4000 /$ monderusse. 10434

ISSN : $1777-5388$

\section{Éditeur}

Éditions de l'EHESS

\section{Édition imprimée}

Date de publication : 1 avril 2018

Pagination : $311-340$

ISBN : 978-2-7132-2746-2

ISSN : $1252-6576$

\section{Référence électronique}

Irina Tcherneva, « Rupture du monopole dans la fabrication du cinéma (1958-1970) », Cahiers du monde russe [En ligne], 59/2-3 | 2018, mis en ligne le 01 avril 2020, consulté le 08 janvier 2022. URL http://journals.openedition.org/monderusse/10434; DOI : https://doi.org/10.4000/monderusse. 10434 


\section{RUPTURE DU MONOPOLE DANS LA FABRICATION DU CINÉMA (1958-1970)}

\section{Studios institutionnels et pratiques économiques alternatives}

L'État soviétique enferma la filière du cinéma dans les bornes d'un trust, qui, dès la fin des années 1930, regroupait production, distribution et exploitation. Le prototype hollywoodien, perçu comme efficace au début de cette décennie par les milieux du cinéma et les décideurs politiques, fut ainsi transposé, bien que fragmentairement, sur le terrain soviétique ${ }^{1}$. En premier lieu, ce trust se caractérisait par le regroupement, au sein d'un même complexe, de l'ensemble des activités (conception, fabrication et édition, vente et diffusion des films). Le mouvement de concentration se déploya aussi à l'horizontale : le nombre de concurrents investis dans le même type de travail fut réduit ${ }^{2}$. Les studios soviétiques concurrents dans les années 1920 (Sovkino, SevZapKino, Vufku en Ukraine, Goskinprom en Géorgie) furent progressivement incorporés au sein d'une structure unique fondée sur le principe de la répartition géographique et de la spécialisation des fabricants de films. Dans le champ de la diffusion, l'intégration horizontale n'empêcha pas cependant la concurrence que représentaient, pour les salles du trust, les clubs ouvriers et les salles dépendant des entreprises. Pour ce qui est de la création, les

1. Au fur et à mesure de la mise en place de ce schéma de concentration, la référence hollywoodienne s'éroda. Notamment les laboratoires et les fabriques productrices de pellicule et de caméras passèrent finalement sous l'administration du ministère de la Défense. Valérie Pozner, «La référence hollywoodienne dans le cinéma soviétique entre 1928 et 1930 » in C. Gauthier, A. Kerlan, D. Vezyroglou, éds., Loin d'Hollywood? Cinématographies nationales et modèle hollywoodien. France, Allemagne, URSS, Chine, 1925-1935, P. - Toulouse : Nouveau Monde Éditions, La Cinémathèque de Toulouse, 2013, p. 54-58. L'incorporation incomplète de la référence états-unienne dans le contexte soviétique est aussi un des fils rouges de l'ouvrage de Maria Belodubrovskaya, Not According to Plan : Filmmaking under Stalin, Ithaca - London : Cornell University Press, 2017.

2. Laurent Creton, Économie du cinéma, P. : Armand Colin, 5édition, 2014 [1994], p. 157-164. 
films réalisés par cette structure s'adressaient à des publics divers, à savoir aussi bien aux spectateurs individuels qu'aux institutions (ministères, entreprises), qui recouraient au cinéma pour promouvoir leurs sites et leurs produits.

Le présent article $^{3}$ étudie comment ce monopole a été rompu, notamment par suite de l'émergence de nouveaux fabricants - les studios institutionnels (vedomstvennye $)^{4}$, financés par des ministères autres que celui du cinéma. Créés auprès des entreprises dans la seconde moitié des années 1950, ces studios émanaient du mouvement du cinéma amateur (kinoljubitel'stvo), au premier abord très éloigné des préoccupations industrielles. Toutefois à la différence des cinémas amateurs européens, puissamment marqués par les pratiques individuelles, leur équivalent soviétique suivit le chemin du fonctionnalisme. Il tendait à devenir le porte-parole des entreprises et à satisfaire à leurs besoins. C'est son activité économique, inconnue dans l'historiographie, qui nous retiendra ici.

L'Union des cinéastes, représentant la corporation cinématographique, qualifiait de non professionnels ceux qui s'investissaient dans la création de films sans avoir de diplôme en cinéma ni faire partie du personnel des studios classiques. Nous démontrerons ici comment ces non professionnels parvinrent à produire et à vendre des films en contournant l'industrie filmique régie par le plan. Envisagés sous l'angle de l'activité non contrôlée par l'administration du film, ces studios constituent un terrain nouveau de la recherche et résonnent avec les travaux sur les pratiques de l'économie informelle ou « de l'ombre $»^{5}$. Nous comptons explorer de la sorte les marges d'autonomie des entreprises soviétiques lors des réformes khrouchtchéviennes et brejnéviennes ${ }^{6}$. À l'aune des recherches menées sur la faculté des acteurs économiques à s'adapter aux problèmes générés par la planification, aussi bien à l'échelle individuelle ${ }^{7}$ qu'à celle des entreprises et des décideurs

3. La rédaction de cet article a profité des échanges fructueux tenus dans le cadre du séminaire franco-russe coorganisé par le CERCEC et l'Institut d'histoire russe de l'Académie des sciences de Russie le 19 octobre 2015. Je remercie chaleureusement les participants à ce séminaire ainsi que Larissa Zakharova et Juliette Cadiot et les relecteurs anonymes des Cahiers du Monde Russe pour leurs critiques et commentaires.

4. Cette dénomination pourrait aussi se traduire par «studio ministériel » ou «studio d'entreprise ». Dans le champ de l'histoire du cinéma, le film institutionnel ou le film d'entreprise désigne une communication filmée interne ou externe d'une société ou d'une corporation.

5. Notamment Alena Ledeneva, Russia's Economy of Favours : Blat, Networking, and Informal Exchange, New York : Cambridge University Press, 1998 ; Richard E. Ericson, " Command Versus "Shadow": The Conflicted Soul of the Soviet Economy ", Comparative Economic Studies, 48 (1), 2006, p. 50-76 ; Gilles Favarel-Garrigues, La police des mœurs économiques : De l'URSS à la Russie, 1965-1995, P. : CNRS, 2007.

6. Retenons en particulier le doctorat de Yakov Feygin, Y. Feygin, « Reforming the Cold War State : Economic Thought, Internationalization, and the Politics of Soviet Reform, 1955-1985», University of Pennsylvania, 2016.

7. Natalya Chernyshova « Consumers as Citizens : Revisiting the Question of Public Disengagement in the Brezhnev Era », in D. Fainberg, A.M. Kalinovsky, éds., Reconsidering Stagnation in the Brezhnev Era: Ideology and Exchange, Lanham : Lexington Books, 2016, p. 3-20. 
économiques ${ }^{8}$, nous montrerons le rapport complexe, marqué par l'interdépendance et par de potentiels conflits, qui s'établit entre la répartition officielle des activités planifiées au sein de l'industrie centralisée du cinéma et les activités marchandes périphériques.

Ces nouveaux studios s'investirent dans la réalisation de films éducatifs, destinés à la formation professionnelle ou informatifs et visant à promouvoir les activités des ministères ou des entreprises auxquels ces studios étaient rattachés. Ce cinéma institutionnel ou utilitaire ${ }^{9}$ était qualifié en URSS de " cinéma de commande » (zakaznoe kino). Un cinéaste en donne un exemple emblématique :

Admettons, le ministère de l'Industrie pétrolière s'occupait de l'exploitation du gisement de Jamal ${ }^{10}$. Il souhaitait faire une proposition au gouvernement, au Comité central du PCUS, etc., et leur présenter les enjeux d'une telle exploitation. Pour ce faire, il commandait un film documentaire. ${ }^{11}$

Tout au long de l'histoire du cinéma, l'administration et, à partir de 1956, les studios classiques placés sous les ordres de celle-ci ont vendu des films commandés par des usines, des ministères, ou des instituts de recherche ${ }^{12}$. Les commanditaires se chargeaient de leur projection auprès d'un public défini. Les studios classiques s'attelèrent à ces commandes, qui constituaient un complément bien rémunéré aux films de fiction et aux documentaires. À la fin de la période stalinienne, ce secteur devint même une source de financement pour l'industrie filmique $^{13}$, car, depuis 1938 , les studios étaient rétribués à chaque réalisation et non pas en fonction des recettes de leurs films. Jusqu'aux réformes khrouchtchéviennes, ce « cinéma de commande » permit à la filière de survivre, tout en contournant l'impératif du succès public : les spectateurs regardaient des films de fiction en partie étrangers, tandis que nombre de studios traditionnels en périphérie fabriquaient des « films de commande », jamais diffusés dans les salles. Avec le redémarrage de la production de longs-métrages de fiction en 1956-1957, ils continuèrent à se financer par ce biais. C'est précisément dans ce secteur stratégique

8. Carole Sigman, « Jeux pervers et charme discret de la planification. Les transformations de la gestion de l'économie dans l'URSS de Kruščev (1962-1964)», in Michel Dobry, Brigitte Gaïti, Johanna Siméant, Assia Boutaleb, éds., La consistance des crises : autour de Michel Dobry, Rennes : Presses universitaires de Rennes, 2018, p. 177-196.

9. Yvonne Zimmerman, et al., Dokumentarische Gebrauchsfilme 1896-1964, Zurich : Limmat Verlag, 2011 ; Charles R. Acland et Haidee Wasson, Useful Cinema, Durham, NC : Duke University Press, 2011.

10. Péninsule située dans le nord de la Russie.

11. Deux entretiens de longue durée avec Vladimir Činënov (réalisateur, au studio traditionnel de Sverdlovsk depuis 1956) - 27 et 28 avril 2009, Ekaterinburg.

12. Spravočnik dokumentov po proizvodstvu hudožestvennyh fil'mov [Recueil de documents sur la production des films de fiction], M. : Iskusstvo, 1958, p. 213-214.

13. Ma thèse de doctorat l'étudie sous l'angle économique, institutionnel et social. Irina Tcherneva, " Le cinéma de non-fiction en URSS : création, production et diffusion (1948-1968) », P. : EHESS, 2014, p. 162-190, 274-292, 615-630. 
du cinéma qu'intervinrent les nouveaux studios non professionnels, appelés aussi institutionnels par leurs détracteurs. Ils mirent en cause l'équilibre de l'industrie filmique planifiée. Le présent article montrera comment les administrations d'État y décélèrent des activités économiques lucratives, auxquelles elles aspirèrent à fixer des limites.

La progression des studios institutionnels dans les années 1960 rejaillit sur la présence traditionnelle, bien que marginale, du cinéma auprès de certains ministères (par exemple, les studios des Commissariats de l'industrie lourde et de la santé depuis les années $1930^{14}$ ). Mais à la fin des années 1950, à la faveur de la déconcentration administrative ${ }^{15}$, puis des réformes économiques menées par Kosygin, les petits studios institutionnels eurent tendance à se multiplier. Les studios classiques étaient alors eux-mêmes tiraillés entre plusieurs projets de réforme qui visaient à répondre à des fléaux permanents, dont une partie n'était pas propre à la production filmique. Il s'agissait notamment du déséquilibre entre les produits fabriqués et leur écoulement, des longs délais de production et des coûts de revient élevés. Au tournant de la décennie 1950, des voix se levèrent pour créer de petites unités autonomes et spécialisées dans certains types de cinéma. Cependant, les studios traditionnels s'orientèrent vers les attentes des républiques et des régions ; ils tenaient à leur garantir des catalogues de films aussi variés que possible. Lors de la réforme Liberman-Kosygin, certaines catégories de personnel de la filière filmique critiquèrent les grandes fabriques de cinéma et les présentèrent comme foncièrement figées et non rentables ${ }^{16}$. À la fin de la période étudiée (fin des années 1970), les studios institutionnels lançaient alors un deuxième défi à la filière. Leurs manières de faire apparaissaient à certains praticiens et aux administratifs du cinéma comme des modèles alternatifs, plus souples et plus efficaces.

La séquence temporelle retenue ici offre donc la possibilité d'étudier une palette de réactions exprimées sur l'activité de ces nouvelles entités par l'administration du cinéma, le Conseil des ministres, le Comité de contrôle du Parti et de l'État ${ }^{17}$, le Comité d'État pour la coordination des travaux de recherche (Gosudarstvennyj komitet Soveta Ministrov SSSR po Koordinacii naučno-issledovatel'skih rabot, GK KNIR $)^{18}$ et par les employés des studios traditionnels. Nous étudierons ici

14. H. Abul-Kasymova, S. Ginzburg, I. Dolinskij, éds., Istorija sovetskogo kino (1917-1967) [Histoire du cinéma soviétique (1917-1967)], M. : Iskusstvo, 1969-1978, t. 2, p. 352-353.

15. Natalya Kibita, Soviet Economic Management under Khrushchev: The Sovnarkhoz Reform, London - New York : Routledge, 2013.

16. Irina Tcherneva, « Imiter le marché, une recette pour le cinéma soviétique ? L'histoire du Studio artistique expérimental (1965-1976) », Cahiers du Monde russe, 54 (3-4), 2014 «En deçà du contrôle idéologique : déploiement des subjectivités, prise en compte des publics», p. 589-621.

17. Renommé en 1965 Comité de contrôle populaire, après une distinction entre le contrôle de l'État et celui du Parti.

18. Créé en mai 1961 et dissout en novembre 1965. Ce comité s'occupait de différentes branches de l'économie, de l'acquisition des équipements étrangers et de la traduction des documents techniques, d'investissements dans les travaux de recherche et de production dans 
trois vagues de contrôle effectué par ces instances en 1963, 1966 et 1970. Pour les examiner à l'échelle républicaine, nous nous appuierons sur les fonds du Comité du cinéma ${ }^{19}$ et du Conseil des ministres de Lettonie ${ }^{20}$. À l'échelle pansoviétique, nous mobiliserons la documentation du fonds du Comité de contrôle du Parti et de l'État ${ }^{21}$, qui contient des données jamais explorées sur la perception, par les instances centrales, de ces pratiques non avalisées. Enfin, des entretiens semidirectifs avec des cinéastes qui ont travaillé dans les deux types de studios, classique et institutionnel, et leurs mémoires nous renseignent sur les rapports plus ou moins officiels entre le champ classique et le champ alternatif de la production de films. Trois temps d'analyse nous semblent indispensables : l'observation du déplacement de l'amateurisme dans le cinéma sur le terrain du cinéma fonctionnel, utilisable par des entreprises, puis l'analyse des vecteurs qui permirent aux studios institutionnels d'empiéter sur le terrain des fabricants traditionnels et d'échapper au contrôle de l'administration du cinéma. Enfin, nous montrerons comment les acteurs de cette administration en vinrent à mettre en doute l'efficacité de la filière filmique planifiée.

\section{L'absorption du cinéma amateur par les institutions}

Le constat de la fabrication de films pour le compte d'institutions industrielles est dressé a posteriori. Lorsque le Comité du contrôle d'État et du Parti, le Conseil des ministres et le GK KNIR lancent une première vérification d'ampleur en 1963, ils ignorent les fondements juridiques sur lesquels s'appuie la création de studios amateurs auprès des entreprises et des institutions économiques, tout comme ils méconnaissent le coût de la production de films, les lieux et la fréquence de leur projection ${ }^{22}$. Le désir de savoir afin de contrôler anime trois vagues d'audits entreprises cette année-là, puis en 1966 et en 1970 à l'échelle pansoviétique. Les dossiers constitués à l'occasion de ces inspections conservent des rapports dressés par des fondateurs ou des financeurs des studios institutionnels, ainsi que des évaluations rédigées par les membres des Comités locaux du contrôle d'État et du Parti (parfois tout à fait positives, nous le verrons). Le GK KNIR intervient aussi au début des années 1960, puis laisse davantage la place à l'administration du cinéma. Les rapports dressés par le bas avancent des informations précises, corroborées par d'autres sources. Dans ces dossiers se déploie une bataille argumentaire entre plusieurs instances qui voient d'un œil bienveillant l'activité des nouveaux

diverses branches. Sachant que le cinéma amateur devait se consacrer à la science et à la technique, le Comité était censé le superviser.

19. LVA (Latvijas Valsts arhīvs - Archives d'État de Lettonie), f. 1405.

20. LVA, f. 270.

21. GARF (Gosudarstvennyj arhiv Rossijskoj federacii - Archives d'État de la Fédération de Russie), f. 9527.

22. GARF, f. 9527 , op. 1, d. 261, 1. 1-2. 
studios. En revanche, la condamnation la plus vive de ces pratiques provient, nous le montrerons, du Comité du contrôle d'État et du Parti à l'échelle centrale. Créé pour scruter les évolutions des ministères, le Comité est violemment opposé, en 1963, à ce phénomène nouveau. À l'issue d'un contrôle, son chef Aleksandr $\breve{S}$ elepin $^{23}$ signe une critique acerbe et sommaire visant plusieurs dizaines de studios non professionnels et, en quelques jours au mois de mai 1963, il parvient à faire discuter la question par le Secrétariat du Comité Central du PCUS et à faire adopter la première interdiction par ordonnance du Conseil des ministres ${ }^{24}$. Dès 1965, le Comité perd pourtant le pouvoir acquis lors de l'attaque menée par Kruščev contre les fiefs ministériels. Cette année-là, le contrôle du Parti et celui de l'État sont de nouveau dissociés ${ }^{25}$. Son deuxième texte législatif rédigé dans le but de freiner la création «des studios institutionnels » date du 24 mars $1966^{26}$. Leur interdiction pure et simple ne semble plus envisagée. Les contrôles ultérieurs interviennent à la manière de coups de frein ponctuels et visent à canaliser les rapports qui se sont noués autour de ce cinéma auquel a recours une myriade d'institutions. Les deux ordonnances prescrivent que l'amateurisme dans le cinéma reste dans le périmètre de la recherche, de la formation et de l'éducation politique. C'est dans cette veine que, dès 1945, le cinéma amateur soviétique en développement a été promu auprès des Maisons de la culture, des syndicats d'usines et des établissements d'enseignement supérieur.

Le film familial, a priori archétype du cinéma amateur, est minoritaire en Union soviétique. En revanche, la pratique des amateurs soviétiques est souvent collective. Dès l'après-guerre, faute de pouvoir acquérir des équipements ${ }^{27}$ et de la pellicule à titre privé, les amateurs trouvent ces ressources auprès des Maisons

23. Chef du KGB de 1958 à 1961, il devient en 1961 secrétaire du CC du PCUS. Sa position de responsable du Comité de contrôle jusqu'en décembre 1965 lui permet d'occuper une place d'importance dans le Gosplan et au sein du Conseil des ministres de l'URSS. Pour plus d'informations sur son activité, cf. l'article de Carole Sigman, « Jeux pervers et charme discret de la planification... ».

24. Décret du Conseil des ministres de l’URSS du 25 juin 1963, \# 720, « De la pratique illicite de la création de studios institutionnels », LVA, f. 1405, op. 1, d. 13, 1. 241-252.

25. Sigman, « Jeux pervers et charme discret de la planification... », p. 190-193.

26. Les arrêtés du Présidium, du secrétariat du Comité central du PCUS, du Conseil des ministres de l'URSS au sujet de « la pratique illicite de la création de studios institutionnels, du développement du cinéma sur la pellicule étroite ». 07.08.1962 - 28.03.1966. Archives du président de la Fédération de Russie, f. 3. op. 35, d. 72.

27. En témoigne la quasi-totalité des Mémoires rédigés par les amateurs. Ceux qui pratiquent le cinéma de manière individuelle achètent des pièces de rechange sur les marchés, pour assembler des appareils. Ainsi, Vladimir Šahov, amateur de Perm' investi dans des cercles de sociabilité qui se forment au marché entre praticiens de la radio et du film, considère que l'orientation des usines d'optique mécanique vers la production militaire a empêché la production de petits équipements pour la photo et le cinéma. Vladimir Šahov, Usypal'nica Kamenskih : poiski $i$ nahodki kinoljubitelja [La chambre funéraire des Kamenskij : les recherches et les trouvailles d'un cinéaste amateur], Perm' : Zvezda, 2003, p. 8. Pour plus d'informations au sujet du nombre de caméras $8 \mathrm{~mm}$ et $16 \mathrm{~mm}$ produites en URSS, cf. Irina Tcherneva, " "À la bonne distance" : investir les espaces délaissés par le cinéma professionnel en URSS depuis les années 1960 », ATALA, 19, 2016 « Passage à l'amateur. Enjeux politiques et esthétiques d'un autre cinéma », p. 140. 
de la culture appartenant au réseau de la Centrale pansoviétique des syndicats (VCSPS), mais aussi auprès des entreprises industrielles et des instituts de formation ${ }^{28}$. Ce rattachement se pérennise pendant la période poststalinienne : les studios amateurs ouvrent grâce aux lignes budgétaires des entreprises. Par exemple, plusieurs graphistes de la Chambre de commerce de Lettonie soviétique fondent leur studio en 1958. Disposant d'un local et de pellicule grâce à la Maison de la culture des syndicats, ils réalisent eux-mêmes une partie des appareils ${ }^{29}$. Souvent des employés d'usines et d'institutions, des techniciens et des mécaniciens s'y retrouvent auprès de leurs employeurs et adaptent, voire fabriquent des équipements. Lorsque l'institution dispose de moyens importants, elle rémunère les filmeurs débutants sur ses fonds et son département d'approvisionnement (otdel snabženija) les fournit en pellicule et en équipement ${ }^{30}$. Parfois, ces petits collectifs récupèrent aussi les anciens équipements des studios professionnels ${ }^{31}$. Ils produisent des films en couleur et en noir et blanc, muets et sonores, en $16 \mathrm{~mm}$ (format considéré comme amateur) et en $35 \mathrm{~mm}$. Leur activité se stabilise dès la fin des années 1950. De la sorte, progressivement, le nombre d'amateurs individuels chute et celui des studios amateurs augmente ${ }^{32}$.

La presse généraliste et cinématographique donne surtout à voir l'investissement citoyen dans une nouvelle activité culturelle autonome, en particulier dans le domaine de la culture et de la science ${ }^{33}$. En effet, les institutions d'enseignement et de recherche prennent part à la création de supports éducatifs et scientifiques filmés. Les unités créées à cet effet apparaissent tantôt comme des studios amateurs tantôt comme des laboratoires de photographie et de cinéma (foto-kino-laboratorii), conformément au décret de $1948^{34}$. Ainsi, à l'échelle de la ville de Sverdlovsk, l'université fédérale d'Oural ${ }^{35}$ et l'Institut polytechnique

28. Jaak Järvine, Vzgljad v prošloe : kratkaja istorija razvitija kinoljubitel'stva v byvšem SSSR $i$ stranah Baltii [Un regard vers le passé : une brève histoire du développement de l'activité cinématographique en amateur en ex-URSS et dans les pays Baltes), Tallinn : OU Vali Press, 2005, p. 20.

29. Entretien avec Osvalds Dinvietis, cinéaste amateur, directeur de l'Association lettone du cinéma amateur, 22 avril 2010, Riga.

30. C'est le cas de l'Institut pansoviétique de recherche en industrie mécanique d'agriculture. GARF, f. 9527, op. 1, d. 261, 1. 28-29.

31. Ainsi, l'ancien équipement inutilisé de Lenkinohronika et Moskinohronika se retrouve dans les mains des amateurs de Iaroslavl' en 1959. GARF, f. 9527, op. 1, d. 261, 1. 56.

32. Selon Jaak Järvine, le nombre de studios atteindra 8000 dans les années 1980. Järvine, Vzgljad v prošloe, p. 11. Les travaux de Maria Vinogradova reviennent également sur ce mouvement d'institutionnalisation. Cf. Maria Vinogradova, « Socialistická filmová tvorba vs. Gosplan: Budování infrastruktury sovětského amatérského filmu », Iluminace, 26 (2), 2016, p. 9-27. Ibid., «Between the state and the kino: Amateur film workshops in the Soviet Union », Studies in European Cinema, 8 (3), 2011, p. 211-225.

33. A. Sokolov, « Kinostudii v vysšej škole [Les studios de cinéma dans une école supérieure] », Iskusstvo kino, $\mathrm{N}^{\circ} 9,1959$, p. 100-101.

34. GARF, f. 9527 , op. 1, d. 261, 1. 78.

35. Entretien avec Arkadij Morozov (réalisateur amateur depuis 1960, puis professionnel, au studio depuis 1966) - mars 2009, Ekaterinbourg. 
ouvrent leurs unités. Les liens entre les milieux scientifiques et industriels s'établissent rapidement. Les amateurs qui travaillent dans ces deux institutions de recherche et d'enseignement (géologues, mathématiciens, ingénieurs chimistes...) entrent en relation avec les studios équivalents créés auprès du comité municipal du Komsomol ${ }^{36}$ et des usines locales. À Sverdlovsk, deux grandes usines, Uralmaš et Kalinin, ouvrent leurs studios ${ }^{37}$. La trajectoire de M. Rafikov, l'un des membres actifs de l'unité cinématographique de l'usine Kalinin, est à l'image de l'inscription des pratiques non professionnelles dans les cercles à la fois industriels, militaires et scientifiques. Il a débuté au syndicat de l'usine Kalinin, qui a acheté des équipements de tournage ${ }^{38}$ pour un groupe de douze personnes. Plus tard, alors qu'il servait dans l'armée, Rafikov s'est allié avec le journaliste militaire Žuravlëv, qui « a toujours rêvé de créer un studio d'actualités cinématographiques, pour imprimer sur pellicule la vie et le travail dévoué des constructeurs militaires $»{ }^{39}$. Ils ont fondé ensemble un studio auprès de la Maison des officiers et ont commencé à produire le ciné-journal intitulé Les Nouvelles de nos jours. Plus tard, Rafikov a mis à profit ces savoir-faire à l'université, où il a créé un studio cinématographique estudiantin URGU ${ }^{40}$-Fil'm.

Il n'est pas rare de voir la naissance de plusieurs dizaines de petites unités d'amateurs dans de grandes villes et régions. De même, il n'est pas rare de voir des ramifications se nouer entre la science, la production, les organisations locales du parti et de l'armée ${ }^{41}$. La création des studios émane souvent d'un accord entre la direction et la commission idéologique de l'institution en question avec les organisations locales du parti ${ }^{42}$. C'est à l'échelle supérieure (républicaine ou pansoviétique) que ces décisions restent méconnues. Dès lors, il est malaisé d'en déterminer le nombre exact. Ainsi, à l'occasion de la première inspection en 1963, un contrôleur écrit :

36. C'est le cas de Gleb Panfilov, futur cinéaste de fiction.

37. Lev Efimov, un des pères fondateurs du studio à Uralmaš, deviendra dans les années 1960 cinéaste employé au studio traditionnel de Sverdlovsk. Lev Efimov, Koe-čto, koe o čëm, koe-kak. Bolee pjatidesjati let $v$ neigrovom kino. Zapiski, vospominanija kinorežissëra [Quelque chose, sur quelque chose, d'une manière quelconque. Plus de cinquante ans dans le cinéma non joué. Notes et souvenirs d'un réalisateur de cinéma], Ekaterinburg, 2011, p. 12.

38. Pour comprendre comment étaient passées ces commandes groupées aux usines produisant des équipements pour le cinéma, il faudrait mener des recherches supplémentaires dans les archives de ces usines.

39. M. Rafikov travaillera par la suite au studio TV de la ville de Sverdlovsk. M. Rafikov, "Ljubov' moja - èlektronnoe čudo [Mon amour - le miracle électronique] », in V. Kostusov, éd., "Govorit i pokazyvaet Sverdlovsk... » Televidenie, kotoroe my delali i ljubili. [« Sverdlovsk transmet... » La télévision que nous faisions et que nous aimions], Ekaterinburg : Bank kul'turnoj informacii, 2005, p. 55-56.

40. Ural'skij gosudarstvennyj universitet [Université d'État d'Oural].

41. À l'instar de l'oblast' de Lugansk où, en 1959, fonctionnent 27 studios. Iskusstvo kino, No. 9, 1959, p. 101.

42. GARF, f. 9527, op. 1, d. 261, 1. 33. 
Dans la république socialiste de Lettonie, il existe 32 laboratoires et studios de cinéma. Ce chiffre est incomplet, puisqu'aucun recensement n'a jamais été mené dans cette république. Les studios ont surgi à des périodes différentes [...], sans autorisation et spontanément (stihijno) suivant le principe : « Si mon voisin en a un, pourquoi pas moi ? $»^{43}$

Cette année-là, on en compte environ 300 en Union soviétique et chaque petite unité réunit de cinq à une vingtaine de membres. Les Maisons de la culture et les établissements de rattachement sont à la fois des lieux de sociabilité pour les amateurs et des espaces privilégiés d'accès aux ressources. En retour, le rattachement institutionnel conditionne les thèmes des films et les objectifs assignés aux amateurs. Ceux-ci répondent aux desiderata de grandes usines et d'institutions pour la création de supports éducatifs, informatifs, de recherche ou promotionnels. C'est ainsi qu'un enthousiaste du cinéma amateur, feuilletant la section dédiée à sa passion de la revue Iskusstvo kino [L'Art du cinéma], peut découvrir que

le studio amateur du combinat de métallurgie de Leninogorsk crée les Annales cinématographiques (Kinoletopis') de sa ville natale. Les films réalisés présentent l'immense centre industriel de l'Altaj durant les premiers jours du septennat et le combat des travailleurs pour l'accomplissement des objectifs du Grand Plan avant l'heure.

Il lira aussi que « le collectif du laboratoire de photographie et de cinématographie de l'usine automobile Lihačëv vient de sortir le sixième numéro du ciné-journal L'Usine automobile de Moscou $»^{44}$. Ces films promeuvent les qualités des entreprises et se trouvent à mi-chemin entre outils de formation professionnelle ou d'éducation civique et supports publicitaires. Le cas du studio du sovnarkhoz de Iaroslavl' illustre bien cette évolution. Créé en 1959 auprès de l'Institut de recherche et voué à tourner des films éducatifs, il commence rapidement à filmer pour les entreprises de la région. De cette façon, il s'autofinance et ne perçoit plus de dotations. Le sovnarkhoz centralise les ressources fournies par ses entreprises et les verse au studio ${ }^{45}$, la capacité du studio à s'autofinancer se révèle cruciale. Un autre laboratoire de photographie et de cinématographie basé à Kiev produit des films pour les écoles techniques : la moitié de son fonds de roulement provient du Bureau scientifique de prospection (Naučno-metodičeskij kabinet), l'autre des commandes industrielles ${ }^{46}$.

Ces films réalisés par le bas, tirés habituellement de trois à sept exemplaires et diffusés à l'échelle de l'oblast ou de l'entreprise, recouvrent une palette d'enjeux. Répondant aux aspirations scientifiques et professionnelles qui animent l'institution

\section{Ibid., 1. 31.}

44. Iskusstvo kino, No. 9, 1959, p. 101.

45. GARF, f. 9527 , op. 1, d. 261, 1. 46-48.

46. Ibid., 1. 86 . 
auprès de laquelle le studio est rattaché, ils ne rejoignent pas forcément la politique définie par l'État pour ce secteur. Par exemple, Progressfil'm de l'Exposition des réalisations de l'économie nationale de l'URSS (VDNH), employant 77 personnes en 1962, consacre plusieurs de ses films aux méthodes d'élevage et à l'implication de scientifiques et de techniciens dans l'agriculture. Quand ce studio passe au crible du contrôle, il s'avère que ces réalisations ne font pas preuve d' " une expérience acquise dans l'industrialisation de l'agriculture $»^{47}$. Ont-elles été d'emblée conçues dans le sillage de l'exploitation intensive et industrielle ? Nous serions également curieux de voir La collectivisation en Ukraine, film commandé par l'Institut d'architecture et de construction de $\mathrm{Kiev}^{48}$. Souvent, les studios dits amateurs portent littéralement à l'écran des ouvertures et des risques (pour l'État) de la décentralisation entamée par Hruščev.

Ce passage, dès la fin des années 1950, des non professionnels d'un cinéma non commercial (éducatif et scientifique) à un cinéma arrimé aux objectifs de certains établissements dérange les contrôleurs centraux. Pour désigner le film institutionnel, ces derniers recourent à des qualifications diverses, telles que film " à caractère publicitaire et informatif " ${ }^{49}$ qui résulterait d'une " aspiration des praticiens du cinéma à s'immortaliser à l'écran avec une série de directeurs exécutifs $»^{50}$. Par exemple, le studio amateur de l'usine DneproSpecStal' tourne un film au titre aussi captivant que Le premier-né $d u 1^{e r}$ quinquennat. Il promeut l'usine, fait la « démonstration du labeur de [ses] travailleurs d'avant-garde » et de dispositifs créés par l'institution tutélaire pour le repos de ses employés ${ }^{51}$. Ce film est projeté 23 fois « dans les ateliers de l'usine, dans les résidences collectives, dans les kolkhozes patronnés par l'usine et dans les régiments militaires, lors de conférences locales du parti et de diverses réunions, ainsi qu'à la télévision de Zaporož'e $»^{52}$. À l'instar d'autres réalisations de ce type, un tel film n'acquiert son sens que localement et contribue intensément à ancrer la légitimité de l'usine ou d'un détenteur local de pouvoir.

Les membres du Contrôle d'État et de Parti qui dressent les procès-verbaux de leurs inspections ne tracent pas de frontière nette entre les « documents cinématographiques (kino-dokumenty) » qui seraient pertinents ${ }^{53}$ et les films utilitaires dérangeants. En revanche, incontestablement, dès lors qu'un film ne s'adresse qu'au public local, ils le jugent « inutile » et dénoncent « une dilapidation des

47. Ibid., 1. 12.

48. Ibid., 1. 78.

49. Ibid., 1. 36.

50. Ibid., 1. 7.

51. En effet, on compte un grand nombre de films consacrés aux maisons de repos et aux camps de pionniers financés par les entreprises.

52. GARF, f. 9527, op. 1, d. 261, 1. 74.

53. Ibid., 1. 28. 
ressources d'État $»^{54}$, bien que le financement soit systématiquement local. Plus encore, l'aspect de la diffusion entre en résonance avec les protestations de certains amateurs contre le virage institutionnel de leur cinéma. Ainsi, en 1967, un membre de la direction du Club moscovite des cinéastes amateurs et responsable du studio auprès de l'usine Kaučuk écrit dans un article :

Pour filmer, on a besoin de pellicule. Pour en acheter, il faut trouver de l'argent. Habituellement, l'entreprise à laquelle est rattaché le studio fournit ces ressources. Mais les dirigeants des entreprises exigent ce qu'ils appellent une « contrepartie », c'est-à-dire la production de films qui n'ont de sens et d'utilité qu'au sein de l'entreprise. Si le studio amateur refuse de produire un tel film, on le met à l'écart et le plus souvent le collectif se désagrège. [...] Actuellement, à Moscou, on compte environ 150 studios amateurs, mais seuls 70 sont « réels », autrement dit créent des films. (...) Les autres ? Certains, ayant acheté de l'équipement, ont fermé, d'autres sont entièrement occupés à produire « des actualités locales $\gg .{ }^{55}$

La parution de cette prise de position n'est guère neutre. Pouvant provenir d'un amateur, elle est tout aussi susceptible de tirer son origine de l'initiative politique, car l'indignation de certains praticiens du cinéma non professionnels est instrumentalisée pour contrôler ces petites unités autonomes.

Ces dernières s'institutionnalisent progressivement et bénéficient d'un appui local incontestable. Leur déploiement est un phénomène peu encadré, souvent ignoré à l'échelle pansoviétique, et résolument nouveau dans le paysage cinématographique. Censé porter l'expression de l'initiative citoyenne, ce cinéma se rapproche en réalité des puissantes institutions de chaque localité. Les créateurs des films sont officiellement employés par ces dernières et leurs marges de manœuvre sont définies par les institutions tutélaires. Une même structure peut aussi bien réaliser un essai lyrique sur les exploits d'une usine (qui patronne localement des activités culturelles) qu'un film promotionnel (produit d'échanges commerciaux et politiques). Il n'est guère aisé de déceler une démarcation entre les deux types de film. Du reste, l'un et l'autre soulèvent les enjeux de la déconcentration administrative et de pratiques économiques qui n’ont pas de précédent juridique.

\section{Le statut du cinéma utilitaire et le risque de briser le monopole central}

Outre la proximité des studios avec leurs financeurs et patrons immédiats (usines, sovnarkhozes, etc.) et, par voie de conséquence, l'absence de garantie que le

54. Ibid., 1. 1-2.

55. Article de M. Baranov, joint au dossier du Comité central du Parti communiste de Lettonie et du Conseil des ministres de LSSR concernant le développement du cinéma amateur, 9 mars 1967, LVA, f. 270, op. 3, d. 3465, 1. 49. Intitulé « Prazdniki i budni malogo ékrana [Les fêtes et le quotidien du petit écran] », il paraît dans la presse pansoviétique en langue russe. 
contenu des films dits amateurs converge avec les orientations du gouvernement et du parti, nous observons aussi que l'amplification et la diversification de la production de ces studios entrent en interférence avec l'industrie filmique. Nous faisons ici l'hypothèse que le Conseil des ministres redoute la création des " films de commande » en dehors de la filière classique, parce qu'il convient de maintenir ce générateur de ressources dans celle-ci. Le " cinéma de commande » est un secteur crucial pour la filière traditionnelle : entre 1956 et 1961, les films commandés et financés par des usines et des ministères constituent une importante source de revenus. En périphérie, où la création de la fiction ne se remet en place qu'au début des années 1960, la part des films de commande oscille entre $50 \%$ et $65 \%$ de la production de toute la non-fiction. En 1962, à l'échelle pansoviétique, ce type de cinéma occupe plus de $50 \%$ de toute la production filmique ${ }^{56}$. Dans la décennie qui suit, un studio traditionnel périphérique peut produire jusqu'à 150 courts-métrages par an, œuvrant ainsi pour la «branche la plus débrouillarde $»^{57}$ sur le plan financier. Tout comme dans d'autres contextes nationaux, le film institutionnel génère des ressources pour le cinéma et se révèle comme moyen de remettre sur pied l'économie du film (à l'instar des films qui visent à promouvoir le plan Marshall en Europe ${ }^{58}$ ). Les réformes khrouchtchéviennes donnent lieu à une distinction plus explicite entre le " film de commande » et le film éducatif ou scientifique, qui poursuit des objectifs d'éducation (prosveščenie) et est diffusé dans les salles publiques. Cependant, ce type de cinéma nécessite un investissement de la part du ministère de la Culture, ainsi que des collaborations pérennes avec les instituts de recherche. Les ministères soviétiques sont peu enclins à agir en mécènes. Dans cette conjoncture, le Conseil des ministres préfère « déléguer » aux studios amateurs le cinéma scientifique et éducatif.

Pourtant, le Comité du contrôle critique aussi les studios professionnels pour leur investissement dans le travail sur commande : dans sa perspective, les cinéastes et les studios se seraient "inféodés » aux commanditaires industriels ${ }^{59}$. Cette critique trouve un écho dans le milieu cinématographique. À cette période, l'Union

56. Note du président adjoint du Contrôle d'État du Conseil des ministres de la RSFSR O. Demjanceva «Au sujet de sérieux défauts dans la production et la diffusion des journaux filmés, des documentaires et des films éducatifs en RSFSR », 25 janvier 1962, RGANI (Rossijskij gosudarstvennyj arhiv noveishei istorii - Archives de l'État russe d'histoire contemporaine], f. 5, op. 37, d. 108, 1. 7 .

57. Vladimir Činënov cité ci-dessus.

58. Noël Van Rens, « Les films du plan Marshall : 1948-1953. La naissance de 1'Europe moderne dans l'image "), in Roger Odin, éd., L'Âge d'or du documentaire. Europe : Années cinquante, P. - Montréal : L'Harmattan, 1998, t. 2, p. 187-226.

59. CDOOSO (Centre de la Documentation des Mouvements Sociaux de la région de Sverdlovsk), fonds du département de la culture et de la science auprès du comité régional du parti de Sverdlovsk, f. 4, op. 54, d. 153, 1. 44-46. Le Contrôle à l'échelle de la Russie soviétique aussi soumet au PCUS, en 1962, un document qui comporte un passage sur la soumission des studios aux besoins des ministères, RGANI, f. 5, op. 37, d. 108, 1. 7. Le Contrôle désigne les films de commande comme « des rapports publicitaires sur le fonctionnement des entreprises et des ministères ». 
des cinéastes et le ministère de la Culture proposent au Comité central du Parti de transférer aux studios institutionnels des ministères de la Défense et de l'Agriculture, une partie du « cinéma de commande » réalisé par les professionnels ${ }^{60}$. Cependant, dès 1962, le ministère de la Culture, puis son héritier, le Comité du cinéma, centralisent les relations avec les commanditaires, sans pour autant établir de frontières claires pour ces relations d'interdépendance. Au contraire, ce « cinéma de commande » est le plus souvent dilué dans la catégorie administrative " cinéma de vulgarisation scientifique ». Cela permet d'alimenter le reste du cinéma en partie par des ressources émanant des entreprises et des ministères. Lorsque divers établissements, usines ou fermes collectives font appel aux studios qui ne relèvent pas de la filière, cet équilibre change.

Les contrôleurs constatent une pratique régulière de la fabrication de films sur commande, qui s'ajoute à d'autres activités rentables pour le champ non professionnel. Ainsi, l'Association des amateurs de cinéma de Lettonie, d'emblée créée en tant qu'organisation autonome sur le plan comptable (hozrasčëtnaja) ${ }^{61}$, se consacre aux arts graphiques, à la production de publicité et d'affiches ${ }^{62}$. En outre, elle réalise annuellement dix films commandés par diverses organisations ${ }^{63}$ dont, par exemple, le court-métrage Panneaux signalétiques réfléchissants ou le film À l'ombre d'un stade (commandé par le stade « Daugava ») ${ }^{64}$. Bien que n'étant pas à but lucratif, l'association ne devait pas être déficitaire ${ }^{65}$. Cependant, lorsqu'en 1966 le Comité du cinéma de Lettonie, le Syndicat pour le cinéma, le studio professionnel et l'Union républicaine des cinéastes contrôlent son activité $^{66}$, ils notent bien que seuls $2 \%$ de son budget proviennent des cotisations des adhérents, tandis que l'Association tire ses ressources financières du développement des pellicules photographiques pour des particuliers, de la vente de cartes postales, ainsi que de la réalisation de diapositives et d'affiches pour des écoles et des musées. Ils considèrent cette activité comme lucrative et expliquent ainsi l'accroissement de l'Association. En effet, à sa création en 1958, l'Association réunissait 155 personnes ; en 1966, ce chiffre était dix fois supérieur. Cette année-là, elle regroupait 26 studios. Selon les rapporteurs, l'Association n'avait pas le droit de réaliser de « films de commande ». Or, ceux-ci représentaient $60 \%$ de sa production ${ }^{67}$.

60. Projet de réforme « À propos des mesures pour un développement de l'art du cinéma en URSS », adressé à Kosygin, 12 mai 1959, RGANI, f. 5, op. 36, d. 78, 1. 79.

61. Ce qui équivaut à la nécessité d'équilibrer les dépenses et les entrées de fonds.

62. Entretien avec Osvalds Dinvietis.

63. LVA, f. 1405, op. 1, d. 13, p. 243.

64. Ibid., 1. 244, 246.

65. Järvine, Vzgljad v prošloe, p. 465.

66. Parmi les membres de l'inspection, Kozlovskis - président du Conseil des syndicats pour le cinéma ; Līcis - président de la section des ciné-amateurs dans l'Union des cinéastes de Lettonie, Pēteris Veselkovs employé du studio classique depuis 1945.

67. LVA, f. 1405 , op. 1, d. 13, 1. 241-252. 
L'activité marchande n'est guère escomptée dans le champ de l'amateurisme et la préoccupation des instances centrales quant à un enrichissement par le bas, contournant la filière, émerge clairement. Fait remarquable, les studios créés par des amateurs peuvent offrir des prix particulièrement attractifs. Ainsi, en moyenne, en 1961, le prix de revient de dix minutes de film est de 2240 roubles; en 1962, il est de 1748 roubles $^{68}$. À la même période, le studio amateur rattaché au sovnarkhoz de la ville de Moscou parvient à proposer des films à la vente pour 5304 roubles ; un film équivalent réalisé par un studio classique coûte 7500 roubles $^{69}$. Cet écart s'explique par le fait que les prix pratiqués dans la filière classique sont définis par le ministère et résultent d'un équilibre complexe dans lequel les taxes prélevées se répartissent entre le documentaire, le film « de commande » et la fiction, la production, la distribution et l'aménagement du territoire en cinéma. Ceux qui défendent leurs studios de proximité ne se privent pas d'insister sur cet atout du cinéma non professionnel. Ainsi, le Bureau central de l'information technique du sovnarkhoz de Volga-Viatka, auquel est rattaché le studio amateur de Iaroslavl', argumente sur le caractère crucial de ce studio de proximité pour l'auto-représentation d'un sovnarkhoz au poids économique si significatif et écrit :

Les entreprises [de la région lui] commandent volontiers des films, car ces derniers sont réalisés en un mois, un mois et demi et coûtent 2500 roubles chacun, ce qui est plusieurs fois moins cher que la production par les studios centraux [autrement dit, professionnels]. Une telle somme est parfaitement acceptable pour les entreprises qui dépensent annuellement plusieurs milliers de roubles pour l'information et la propagande techniques. ${ }^{70}$

Cette concurrence entre le domaine étatique et le domaine privé en matière de prix mérite d'être soulignée, car, tout en étant les hérauts de responsables locaux, les studios institutionnels semblent fort habiles dans la relation « de la demande et de l'offre », contrairement à leurs homologues traditionnels.

L'idée de la concurrence spécifique émerge graduellement. Au sein d'une abondante documentation générée par les contrôles, une revendication en particulier retient notre intérêt. Le directeur du studio institutionnel auprès du sovnarkhoz de la ville de Moscou met le doigt sur des conflits potentiels, il souligne que le décret du 28 novembre 1938, à l'origine du monopole du ministère du Cinéma sur la production des films, constitue une entrave au fonctionnement de structures comme la sienne. Il invoque finement les atouts de la décentralisation récente dans la gestion économique ${ }^{71}$. En effet, au fur et à mesure du déploiement des studios étudiés ici, la filière du cinéma - ministère de la Culture, puis le Comité du cinéma

68. GARF, f. 9527 , op. 1, d. 261, 1. 52.

69. Ibid., 1. 64.

70. Ibid., 1. 47.

71. Ibid., 1. 69 . 
- risque de voir son monopole brisé. En cela, la situation des années 1960 est, sans conteste, inédite.

Dès l'audit de 1963, la Pravda du 25 avril 1963 publie un article intitulé « Derrière les écrans des « ciné-artels » institutionnels [Za èkranami vedomstvennyh "kinoartelej"] », qui est une version condensée du rapport de synthèse établi par un certain Sisnev, directeur adjoint du Comité du contrôle des instituts de recherche, sur la base de rapports envoyés par les oblasts et les sovnarkhozes. Ce rapport, signé par Šelepin, renverse complètement les données fournies par le bas. Il affirme ainsi que le prix coûtant des films « de commande » amateurs est bien plus élevé que celui des studios professionnels ${ }^{72}$. Aucun document venant des localités étudiées ne le corrobore. Ce rapport et l'article sonnent le glas de Progressfil'm mentionné plus haut et choisi comme bouc émissaire. Désormais, un studio « classique » doit l'absorber ${ }^{73}$. L'article et le rapport déclenchent par ailleurs plusieurs réunions de mea culpa et quelques lettres d'une indignation orchestrée. Ainsi, les employés du studio de Kujbyšev dénoncent « les nombreux studios de cet acabit qui pullulent dans le territoire desservi par [leur] studio », tout comme ils vilipendent leurs propres collègues « qui ont changé de camp (perebežali), attirés par des salaires démesurément élevés $»^{74}$. Ce vocabulaire belliqueux et politiquement clivant $^{75}$ ne fait que souligner l'instrumentalisation de cette lettre par le Comité du contrôle. On y dénonce même la détention d'équipements étrangers par des amateurs indignes. Le caractère concurrentiel de ces studios mis en évidence dans cette lettre rejoint la perception qu'a de la situation le Comité du contrôle. Nonobstant, les retours de professionnels que j'ai pu réunir ne témoignent guère de cette perception compétitive. Il est révélateur que ce soient « les employés » et non l'administration du cinéma à l'échelle centrale qui soient les signataires d'une telle lettre. Les cinéastes professionnels et les studios, contrairement à l'administration, ne sont nullement touchés par une telle « concurrence déloyale», et pour cause...

72. Ibid., 1. 92.

73. Ibid., 1. 15-16.

74. Ibid., 1. 112.

75. Que l'on retrouve dans les attaques du marché privé entamées en 1948, après une phase de tolérance des autorités fiscales envers les activités marchandes privées. Julie Hessler, « A Postwar Perestroika ? Toward a History of Private Enterprise in the USSR », Slavic Review, 57 (3), 1998, p. 533-536. 


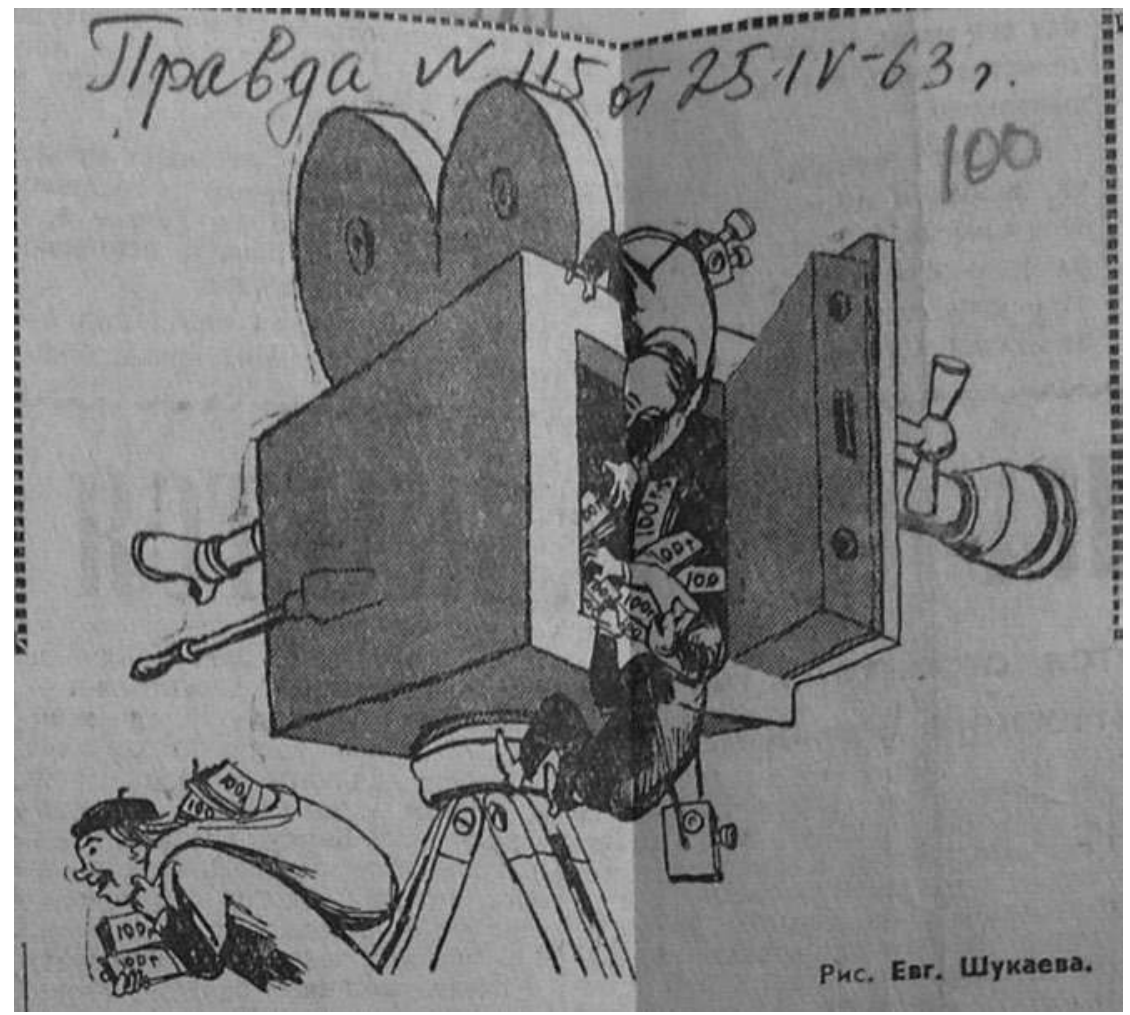

Article de la Pravda, 25 avril 1963, dessin de E. Šukaev

\section{S'aventurer aux frontières du permis. Fixer les bornes de la révision de la filière}

Outre les enjeux propres au renouvellement du paysage cinématographique, les audits mettent au jour des transactions financières et des pratiques qui innervent la production filmique et les industries techniques. Il est révélateur d'ailleurs que, progressivement, le GK KNIR laisse sa place de contrôleur aux structures administratives du cinéma, concernées au premier chef par les pratiques non planifiées dans la filière. Les Conseils des ministres y jouent aussi un rôle crucial, car tout au long de la période étudiée, les inspections révèlent des transactions plus ou moins coordonnées par le bas, en dissonance avec la planification conçue au centre.

Certains laboratoires et studios parviennent à s'autofinancer, mais leurs membres trouvent aussi dans la pratique non professionnelle la possibilité de s'investir dans des activités économiques rémunératrices. Les auteurs des scénarios (internes ou externes à chaque studio) touchent entre 50 et 300 roubles par script. Des contrats sont également conclus avec des compositeurs, des régisseurs du son, des commen- 
tateurs, des animateurs, conformément aux ordonnances de 1950 et $1952^{76}$, qui avaient redéfini la rémunération des agents contractuels par les studios professionnels. La sonorisation de 80 minutes de prises de vue est facturée 120 roubles. Le rédacteur perçoit 100 roubles par mois, le réalisateur-opérateur 140, 160 ou 170 roubles $^{77}$. Les personnes concernées par ces versements sont majoritairement des professionnels issus d'autres domaines que le cinéma. Les salariés des studios non professionnels (lorsqu'il y en a) sont rémunérés directement par leurs institutions de rattachement. Leurs payes varient en fonction des établissements, des oblasts et des républiques : elles ne correspondent pas aux grilles tarifaires de la filière filmique. Ainsi, une inspection menée en 1966 par les contrôleurs républicains à Riga met au jour la disparité de fonctionnement entre les studios amateurs et l'industrie traditionnelle du film. Cette fois-ci, les évaluateurs représentent massivement le Comité du cinéma, le Syndicat des travailleurs de la culture, l'Union républicaine des cinéastes, directement intéressés par la commande régulière de films par les ministères. Ils mettent en exergue un problème essentiel : leurs instances « n’ont jamais approuvé les contrats, la réalisation, la rémunération, le contenu des films » amateurs créés par l'Association des cinéastes amateurs de la Lettonie ${ }^{78}$. De surcroît, leur audit révèle que cette association rémunère ses membres mieux que la loi ne le permet. Elle n'applique pas de grilles tarifaires, pas plus qu'elle n'échelonne les films par catégorie et, partant, elle contourne les règles de l'activité professionnelle ${ }^{79}$. L'opacité dans les transactions financières et dans la rémunération revient constamment dans ce rapport d'expertise et le Comité républicain du cinéma aspire à y mettre fin.

En outre, nous pouvons observer un mouvement général : certains cinéastes salariés des studios classiques abandonnent leur maison mère pour aller travailler dans les studios institutionnels, attirés par une rémunération plus importante et de meilleures possibilités d'évolution de carrière ${ }^{80}$. Par exemple, sur cinq employés du studio, créé en 1959 auprès de l'Institut de recherche du sovnarkhoz de Iaroslavl', trois ont un diplôme d'études supérieures en cinéma, quatre ont une expérience de travail dans les studios cinématographiques et télévisuels de Sverdlovsk, de Rostov et de Volgograd ${ }^{81}$. Les chiffres qui illustrent ces mouvements de personnel apparaissent ici et là, dressant un tableau d'un flux constant.

Qui plus est, diverses sources révèlent des coopérations entre le champ amateur et le champ professionnel. Par exemple, en 1960, un cinéaste employé du studio

76. GARF, f. 9527 , op. 1, d. 261, 1. 53-55.

77. Ibid., 1. 66-68.

78. LVA, f. 1405, op. 1, d. 13, 1. 246-247.

79. Tandis que le nombre de copies tirées, les modalités de diffusion et le payement des réalisateurs et des opérateurs dépendent de ces catégories.

80. Un tel va-et-vient se produit entre le studio professionnel de Sverdlovsk et le studio d’Uralmaš. Entretien avec Vladimir Putincev (opérateur, au studio depuis 1964) - 27 avril 2009, Ekaterinburg.

81. GARF, f. 9527 , op. 1, d. 261, 1. 46-49. 
traditionnel de Sverdlovsk, Aleksandr Litvinov, et les membres du studio amateur d'Uralmaš réalisent ensemble un film de commande sur l'usine. Litvinov agit en tant qu'intermédiaire et apporte une aide professionnelle et technique. Le développement de la pellicule, la composition de la bande-son, le montage du film sont exécutés par le studio professionnel de Sverdlovsk. Cette collaboration se poursuit, comme en témoigne Lev Efimov, amateur au studio d'Uralmaš :

Litvinov me propose : «J'ai besoin de faire un petit film de commande sur un thème lié à ton usine. Faisons-le ensemble : tu vas filmer, je t'aiderai. On partagera l'argent gagné. » Bien entendu, j'ai accepté. Tout était non officiel. Je suis allé à Nižnij Tagil [ville industrielle voisine] avec un opérateur du studio [classique], nous avons filmé. J'ai tout monté dans la foulée. Litvinov a regardé le film pour en corriger certains aspects. Et c'était prêt. ${ }^{82}$

Les flux financiers qui sous-tendent cette coopération sont masqués dans les archives du studio professionnel. La collaboration elle-même trouve ses origines dans l'insuffisance de l'équipement des studios non professionnels. Rares sont ceux qui peuvent prendre en charge toute la chaîne de la production, à l'instar de celui de l'usine automobile de Gor'kij qui, d'ailleurs, parvient à s'autofinancer dès $1957^{83}$. Pour sonoriser les prises de vue et tirer les copies, la plupart se tournent vers leurs homologues classiques de leur localité, bénéficiant ainsi du réseau territorialisé de la filière filmique. Il en est ainsi de l'usine Uralmaš avec le studio de Sverdlovsk, des amateurs de Riga, qui concluent des accords avec les professionnels dans leur ville, ou encore du studio du sovnarkhoz Volga-Viatka qui recourt régulièrement aux services des studios classiques de Rostov et de Saratov ${ }^{84}$. Les contrats pour prestation de services (lorsqu'il y en a) sont établis avec les travailleurs des studios classiques et de la télévision et avec les commanditaires industriels $^{85}$. Des coopérations informelles se pérennisent aussi. Cela représente un défi pour les instances centrales. En effet, lorsque de tels travaux s'effectuent à l'amiable et n'apparaissent pas dans les lignes de dépense de leurs studios employeurs, les dépenses en ressources matérielles et en temps de travail sortent du périmètre du plan économique de leur propre entreprise ${ }^{86}$. Les collaborateurs des amateurs utilisent les techniques, les locaux et associent leurs collègues techniciens à ce travail ponctuel, contractuel, réalisé pour des commanditaires externes. Prenons aussi en compte l'impossibilité d'évaluer le temps de travail passé sur ces projets filmiques par les techniciens et ingénieurs de ces studios. Au cours de la troisième phase du contrôle des studios institutionnels, en 1970, leur interférence avec la

82. Efimov, Koe-čto, koe o čëm, koe-kak, p. 19.

83. GARF, f. 9527 , op. 1, d. 261, 1. 37.

84. Ibid., 1. 21-22 et ob, 50.

85. GARF, f. 9527 , op. 1, d. 3034, 1. 75.

86. Ibid., 1. 36. 
planification s'est suffisamment amplifiée pour se trouver au cœur du débat. Cette année-là, le rapport du Comité de contrôle signale :

Les commandes des entreprises de cinéma sont devenues des sources de « gain facile » pour de nombreux employés des studios [professionnels] de cinéma et de TV. Le Studio du comité exécutif de Moscou [studio non professionnel], à lui seul, a versé l'année dernière 40000 roubles de rémunération sur contrat. (...) Plus de 80 salariés de Mosfil'm ont exécuté, sans en informer leur direction, divers travaux pour les entreprises susmentionnées. Ils ont touché leur rémunération directement, ont utilisé à leur guise les locaux et l'équipement de Mosfil'm [studio classique], ont puisé dans les réserves d'énergie et de matériaux du studio. De même, 70 fonctionnaires du Studio pansoviétique de la radio et de la télévision et plus de 60 travailleurs du studio TV de Leningrad ont effectué des travaux sur contrat. ${ }^{87}$

Nous pouvons ainsi observer sur le terrain du cinéma un phénomène rencontré dans d'autres secteurs de l'économie soviétique : les employés des entreprises mettent les ressources de l'institution de leur rattachement officiel au profit d'un travail parallèle. Même lorsque les studios classiques locaux établissent des factures avec les non professionnels, ces échanges monétisés restent méconnus des décideurs centraux. Ainsi, face au Comité du contrôle d'État, le directeur du Studio de la publicité radiophonique et télévisuelle auprès du comité exécutif de Moscou [studio non professionnel] « est obligé de reconnaître, sans tourner autour du pot, que son studio sert d'intermédiaire » entre les professionnels et les commanditaires. Il s'apparenterait donc à une coquille vide, dont la fonction précise est celle d'un prestataire. La même inspection révèle que les salariés du Studio central du cinéma documentaire (CSDF), de Mosfil'm et des studios de Riga et de Leningrad qui participent à ces transactions constituent peu à peu des réseaux de collaborateurs qui vont bien au-delà de leurs employeurs immédiats. C'est précisément pour cette raison que les professionnels et les studios classiques ne perçoivent pas leurs confrères amateurs comme des concurrents, contrairement à la direction de la filière.

Un deuxième enjeu consiste en la nécessité de trouver, au sein de l'industrie planifiée, de la pellicule et de l'équipement. Même si les institutions finançant leurs unités filmiques en achètent aux industries techniques du cinéma, la façon dont elles le font reste plus ou moins nébuleuse, plus ou moins contrôlée. Par exemple, entre 1961 et 1963, à Riga, l'usine VEF qui abrite un studio amateur a reçu 9699 mètres de pellicule, via les instances moscovite et riganaise d'approvisionnement (Kul'tsnab), du Magasin central de Riga et de la fabrique $\mathrm{N}^{\circ} 5$ de Iaroslavl ${ }^{\prime 88}$, tandis que le Département d'approvisionnement de Lettonie (Snabsbyt) fournit annuellement de 3500 à 4000 mètres de pellicule au petit studio amateur auprès de RigaSel'Maš $^{89}$. Quant au studio du sovnarkhoz Volga-Viatka, ses 302000 mètres

87. Ibid., 1. 86.

88. GARF, f. 9527 , op. 1, d. $261,1.32$.

89. Ibid., 1. 34. 
de pellicule dépensés de 1959 à 1963 ont été fournis grâce aux accords avec le Département d'approvisionnement des ministères de la Culture de l'URSS et de la RSFSR. Les contrôleurs de 1963 s'interrogent pourtant sur les stocks conservés par le studio durant cette année-là ${ }^{90}$. Ces courroies d'approvisionnement sont donc légalisées, mais à petite échelle. L'administration centrale du cinéma les découvre, à son tour, progressivement et de façon parcellaire. En 1963, le rapport du Comité de contrôle spécule sur les chiffres disponibles parlant de « millions de mètres de pellicule, suffisants pour créer plusieurs dizaines de longs-métrages de fiction ${ }^{91}$, faisant de nouveau usage de l'argument idéologique hostile à l'initiative d'en bas. Malgré l'incertitude sur les chiffres, la tendance est réelle et l'administration centrale du cinéma aspire à garder ces denrées rares au sein de la filière qui fonctionne selon le plan.

Ajoutons que les équipements que manient les amateurs les familiarisent avec les formats proprement professionnels : $35 \mathrm{~mm}$ à la place du $8 \mathrm{~mm}$ ou du $16 \mathrm{~mm}$. De ce fait, les circuits techniques ne sont pas complètement séparés ${ }^{92}$ et cela facilite les collaborations entre professionnels et non professionnels. Pour contrer cet entremêlement des pratiques amateurs et professionnelles, des appels plus ou moins explicites à mettre à disposition des équipements en formats $8 \mathrm{~mm}$ et $16 \mathrm{~mm}$ sont souvent publiés ${ }^{93}$, mais ceux-ci ne seront accessibles qu'au début des années $19700^{94}$. Dans cette veine, tout en dénonçant en 1966 l'activité économique de l'Association des cinéastes amateurs de Lettonie, le Comité du cinéma de la république postule sa reprise en main, du point de vue financier par son administration, et du point de vue professionnel par un centre interrépublicain, " appelé à fournir des services techniques et artistiques aux cinéastes amateurs $»^{95}$. Les professionnels du film sont mobilisés pour encadrer, canaliser et remettre les amateurs sur le droit chemin de l'investissement civique dans les activités artistiques ${ }^{96}$. L'Union des cinéastes propose de créer une nouvelle association et de coopter les membres employés avec le Conseil des syndicats, le Comité du cinéma et la centrale syndicale de Lettonie (LPSPS). Désormais, c'est l'ex-directeur du département des actualités au studio

90. Ibid., 1. 40-42.

91. Ibid., 1. 90.

92. Les studios d'Uralmaš, du Komsomol et de l'Institut polytechnique réalisent des films sonores en $35 \mathrm{~mm}$. Efimov, Koe-čto, koe o čëm, koe-kak, p. 12. L'administration du cinéma cherchant à valoriser l'investissement des amateurs (tout en lui créant un espace distinct) met en place des festivals spécifiques. Ce cadre est révélateur des formats utilisés. Ainsi, en 1962, $80 \%$ des films projetés au festival pansoviétique du cinéma amateur sont en $35 \mathrm{~mm}$. Järvine, Vzgljad v prošloe, p. 36.

93. Iskusstvo kino, $\mathrm{N}^{\circ} 5,1958$, p. 151; S. Antonov, «Pervye itogi, pervye razdum'ja... [Premier bilan, premières réflexions...] », Iskusstvo kino, $\mathrm{N}^{\circ} 8,1959$, p. 135-137.

94. Šahov, Usypal'nica Kamenskih, p. 11.

95. Lettre du Comité du cinéma de Lettonie au Comité central du Parti communiste de Lettonie et au Conseil des ministres de la république, 9 mars 1967, LVA, f. 270, op. 3, d. 3465, 1. 45.

96. Lettre du Comité républicain du cinéma adressée au Comité central du Parti communiste de Lettonie et au Conseil des ministres de Lettonie, 9 mars 1967, LVA, fonds du Conseil des ministres de la LSSR, f. 270, op. 3, d. 3465, 1. 38-47. 
professionnel qui la supervise. Enfin, l'Association acquiert un local au centre de la ville pour projeter ses films ${ }^{97}$. Paradoxalement, cette action du Comité illustre à la fois un rétablissement de la primauté du corps de métiers et un acquiescement à la professionnalisation des amateurs.

Tout comme pour d'autres studios institutionnels, l'activité de l'Association des cinéastes amateurs de Lettonie n'était pas méconnue des autorités locales. À sa création, officialisée par le Conseil des ministres de la république, des photographes professionnels s'y sont investis, qui souhaitaient prendre leurs distances avec les ateliers d'État. Jusqu'en 1966, c'est un employé du comité exécutif du parti du district qui dirige cette institution et c'est le président adjoint du Comité du cinéma qui supervise son travail ${ }^{98}$. L'activité de l'Association ne peut donc pas être ignorée de ces acteurs qui la « jugent» en 1966. En revanche, son contrôle révèle un changement de perception. Tout d'abord, à l'échelle pansoviétique, l'activité des studios institutionnels semble prendre de l'ampleur dans la seconde moitié des années 1960. Puis, un nouveau coup de frein pansoviétique constitue un tremplin pour le Comité républicain du cinéma, qui peut réagir aux logiques d'enrichissement par le bas ou de contournement des studios classiques. Si, en 1963, le Comité du contrôle d'État et du Parti parvient à imposer sa sentence répressive au GK KNIR, aux sovnarkhozes et aux membres du Contrôle à l'échelle des oblasts, en 1966 en Lettonie, la situation diffère. Cette fois-ci, le Comité du cinéma républicain s'appuie sur l'incitation du Conseil des ministres de l'URSS à sortir les collectifs amateurs de leur dépendance vis-à-vis des organismes financeurs. À cet effet, la résolution de 1966 prévoit de développer une infrastructure destinée aux cinéastes amateurs (magasins spéciaux, bureaux de distribution, ateliers de réparation). ${ }^{99}$ Toutefois à l'échelle pansoviétique, le mouvement se maintient. Ainsi l'audit de 1970, moins politisé que le contrôle de 1963, révèle que les fabriques de pellicule à Šostka et Kazan' continuent à vendre leur production en dehors de leur plan ${ }^{100}$. Le Contrôle déclare cette vente illégale et le président de la

\section{Ibid., 1. 47.}

98. Järvine, Vzgljad v prošloe, p. 464-465.

99. « Du développement du cinéma sur pellicule étroite et de son utilisation à des fins éducatives et politiques $\gg$.

100. GARF, f. 9527, op. 1, d. 3034, 1. 81-82. Les documents mentionnent 174000 mètres de pellicule vendus annuellement par ces deux fabriques en dehors du plan. Il est difficile de mesurer la part que la pellicule vendue aux ministères occupe dans le volume global produit. Le volume prévu pour 1965, par exemple, équivaut à 1,9 million de mètres (LVA, f. 678, op. 1, d. 204, 1. 67). Ce dernier chiffre comprend pourtant la pellicule négative (pour le filmage), la positive (pour tirer les copies destinées aux salles) et la pellicule destinée à l'armée. Très probablement, seul le négatif était vendu aux studios institutionnels. Le nombre indiqué dans les documents du contrôle témoignerait dès lors d'une part plutôt marginale, à condition que les fabriques de pellicule ne l'aient pas artificiellement baissé lors du contrôle. Les studios professionnels, eux aussi, participent à ce marché parallèle pour obtenir cette denrée rare auprès des fabriques. Ce phénomène, déjà présent dans les années 1930, s'était accentué dans l'après-guerre. Valérie Pozner l'observe pour les années 1946-1947 (RGALI, f. 2456, op. 1, d. 1451, 1. 13). 
Direction principale de l'industrie photochimique interdit ce commerce et impose des amendes aux deux fabriques.

Non seulement les studios qui sont contrôlés n'agissent pas dans l'ombre, mais aussi ils sont pris dans des relations économiques avec les ministères républicains et pansoviétiques, à qui ils vendent des films ${ }^{101}$. Si les administrations du cinéma au niveau des républiques et de l'URSS aspirent à encadrer leur activité, d'autres ministères recourent amplement à leurs services. Les organisations locales du parti et les Conseils républicains des ministres leur servent d'appui. Les textes législatifs et les inspections qui en découlent signalent en creux que ce cinéma non professionnel menace d'entrer en concurrence avec l'industrie filmique. Ils révèlent aussi que le plan de la filière cinématographique (industries techniques, production, diffusion) est dépassé, au profit de diverses formes de transactions marchandes dans lesquelles sont impliqués les professionnels du cinéma ${ }^{102}$. L'introduction de la souplesse dans le système pousse en effet à l'autonomisation des activités rémunératrices par rapport au plan ${ }^{103}$. Celles-ci en viennent à mettre en question les bases économiques du système officiel établi et suscitent une réaction politique hésitante.

\section{De la répression, via l'encadrement, vers une mise en cause des pratiques orthodoxes}

Nous avons déjà souligné le caractère systématiquement parcellaire des informations dont disposent les contrôleurs. L'apparition des studios en dépit des interdictions formelles de 1963 et 1966 s'explique par des ententes locales et une faible application des sanctions pénales ${ }^{104}$. En outre, la réaction des autorités dépend de la configuration des instances impliquées dans les audits et de leur poids politique. Les documents émanant des inspections révèlent des transactions marchandes qui contournent le système planifié, mais aussi constatent la stabilisation progressive des studios institutionnels. Leur mode de fonctionnement met en cause l'efficacité même de l'industrie filmique planifiée. À cet égard aussi, l'administration du cinéma et la communauté professionnelle réagissent différemment. L'inspection menée entre juin et septembre 1970 nous en fournit une illustration parfaite, quand le Contrôle passe au peigne fin les laboratoires de film de Riga, de Vilnius, de Leningrad, ainsi que le combinat (kombinat) des arts appliqués de

101. LVA, f. 1405, op. 1, d. 13, 1. 241-252. GARF, f. 9527, op. 1, d. 3034, 1.63.

102. Plusieurs études offrent des reconstructions analytiques de ces pratiques à l'échelle individuelle, pour l'obtention de produits rares : Larissa Zakharova, S'habiller à la soviétique : la mode et le Dégel en URSS, P. : CNRS, 2011 ; Chernyshova, "Consumers as Citizens », p. 3-20.

103. À l'instar de l'entrepreneuriat privé développé dès 1987 au sein des entreprises d'État sous la dénomination de coopératives.

104. On en trouve des exemples dans d'autres domaines de production, comme le souligne Carole Sigman dans son article « Jeux pervers et charme discret de la planification... », p. 183. 
Lettonie (SojuzTorgReklama), le studio de la Direction pour l'approvisionnement (Gossnab) de Lettonie, le combinat fabriquant la publicité des produits alimentaires (RosPiščPromReklama), le studio de la publicité radio et TV auprès de la Direction municipale du design et de la publicité du comité exécutif du parti de Moscou, le combinat TelePressTorgReklama et le studio ReklamFil'm d'Estonie. Tous ces studios sont institutionnels.

La particularité de cet audit tient à l'attention portée à un secteur ayant connu une croissance récente, la publicité filmée de marchandises. Appelée à devenir un secteur clef, la réclame filmée attire fortement l'attention du Contrôle d'État et de Parti. Ses représentants appréhendent aussi bien la publicité « des résultats » dont les usines souhaitent faire la démonstration aux autorités, à leurs homologues ou aux délégations étrangères ${ }^{105}$, que l'incitation à acheter des produits concrets. Le premier point semble superflu aux contrôleurs, qui incitent les amateurs à se consacrer aux thèmes d'innovation technologique et à la transmission des savoirs professionnels. L'intérêt pour le second émerge progressivement. Des studios rattachés à des sovnarkhozes, au VDNH, à l'Académie pansoviétique d'architecture et de construction fabriquent ces films publicitaires en quantité. Lorsque les contrôles antérieurs à 1970 identifient que les films non professionnels promeuvent des marchandises (voitures, produits cosmétiques), le Comité postule que « la publicité doit être produite exclusivement [...] par les studios spécialisés en cinéma documentaire $»^{106}$, autrement dit par les professionnels. Cependant, il est difficile d'endiguer le mouvement amorcé. Malgré les interdictions, nous notons ainsi la création en 1967 à Tallinn du studio non professionnel ReklamFil'm (ERF) qui, quelque trois ans plus tard, réalise près de 160 films par an. D'une durée d'une à deux minutes, ces films font aussi bien la publicité de la vaisselle que celle de produits cosmétiques. La réclame de produits de consommation brasse des intérêts financiers qui se concrétisent au fur et à mesure que la société soviétique se tourne vers la consommation. Vers 1970, l'inspection met en évidence le caractère indispensable du film publicitaire, ce qui en fait un produit recherché. Cette année-là, le ministre adjoint du Commerce de l'URSS, P. Gorbačëv, fait valoir auprès du Comité de contrôle populaire ${ }^{107}$ qu'il faut voir en l'absence de promotion l'une des raisons de la mauvaise distribution des marchandises fabriquées en Union soviétique. ${ }^{108}$ Le Comité du cinéma est informé du désir des ministères de faire de la réclame filmique pour leurs produits, mais son plan de production ne le prévoit que marginalement. En retour, les commanditaires ministériels des films, ces consommateurs singuliers, rendent visible la concurrence entre la filière classique et les studios institutionnels. Ils reprochent à l'administration du cinéma ses incessantes vérifications qui freinent

105. GARF f. 9527, op. 1, d. 261, 1. 32.

106. Et ce, dès 1963, GARF, f. 9527 , op. 1, d. 261, 1. 7-8.

107. Nouveau nom du Comité de contrôle d'État et de Parti. Cf. note 17.

108. GARF, f. 9527 , op. 1, d. 3034, 1. 12-14. 
la production. En contrepartie, ces mêmes commanditaires, au sein des mêmes plaintes, invoquent les atouts déjà cités des studios institutionnels (prix et délais de fabrication $)^{109}$. Enfin, la publicité produite par les studios institutionnels connaît une meilleure diffusion que celle de leurs homologues traditionnels : en 1969, sur l'ensemble de la publicité diffusée par la TV centrale, dix-huit heures ont été produites par le studio institutionnel TelePressTorgReklama contre quatre par le studio classique de la publicité radio et $\mathrm{TV}^{110}$.

Cette compétition dans un champ filmique nouveau s'accentue par le constat que, vers la fin de la décennie 1960, les studios institutionnels semblent s'être professionnalisés $^{111}$. Tout au long des années 1960, nous y observons la création de postes stables. Les méthodes de travail ne sont plus artisanales, les cinéastes ont acquis une expérience. Ainsi, en 1970, deux évaluateurs, employés du studio professionnel CentrNaučFil'm et qui réalisent l'audit aux côtés de l'administration du cinéma et du Contrôle populaire, constatent « la bonne qualité professionnelle du point de vue de la réalisation et de la prise de vues » des films de plusieurs studios institutionnels ${ }^{112}$. Les experts, la directrice de la rédaction des films publicitaires V. Šilova et le rédacteur O. Krutov, soutiennent que la qualité des films publicitaires créés par le studio institutionnel d'Estonie est même meilleure que celle des films réalisés par leur propre collectif. Cette évolution réelle est due, en premier lieu, à la formation dispensée par les professionnels du cinéma aux non professionnels qu'ils patronnent, mais aussi à la participation ponctuelle des personnels des studios classiques à la fabrication des films par les studios institutionnels.

$\mathrm{Si}$, en 1963, l'absorption de certains studios institutionnels par leurs homologues professionnels s'apparente à une répression, en 1970, lorsque cette décision est prise, c'est qu'on y trouve une autre utilité. Il s'agit d'une tentative de mettre les pratiques de la sphère non professionnelle au service du système classique afin de le dynamiser. Cela se traduit par la fermeture de plusieurs studios institutionnels producteurs de films publicitaires des ministères, ou l'interdiction pour d'autres de réaliser ce type de films. Les dirigeants de ces studios et des ministères auprès desquels ils agissaient sont sanctionnés par des amendes pour « de nombreuses violations de la discipline financière » et reçoivent des blâmes. Toutefois, le studio EstReklamFil'm d'Estonie déjà mentionné continue à fonctionner et

le Comité du Cinéma informe qu'il est en train d'élaborer des propositions pour baisser le coût et réduire les délais de production de films commandés par le

109. Ibid., 1. 78-79.

110. Ibid., 1. 53.

111. Phénomène également observé pour d'autres sphères d'activités. Cf. Igor' Narskij, " Meždu sovetskoj gordost'ju, političeskoj bditel'nostju i kul'turnym šokom. Amerikanskie gastroli narodnogo ansamblja tancev «Samocvety» v 1979 godu [Entre fierté soviétique,vigilance politique et choc culturel : la tournée américaine du groupe populaire de danse "Samocvety"] », Cahiers du monde russe, 54 (1-2), 2013, p. 329-351.

112. GARF, f. 9527, op. 1, d. 3034, 1. 54. 
ministère du Commerce de l'URSS. Les studios [professionnels] ont trouvé la possibilité de faire entrer dans leur plan de production des films sur commande d'organisations commerciales. ${ }^{113}$

En ce qui concerne le studio institutionnel de publicité radiophonique et télévisuelle dépendant du comité exécutif de Moscou, la filière professionnelle l'incorporera franchement parce qu'elle « ne possède pas d'unités spécialisées en création de publicité $\rangle^{114}$. Les audits incitent donc la filière cinématographique à modifier son fonctionnement afin de satisfaire les besoins des institutions, faute de quoi celles-ci recourent à la production filmique hors plan.

Dans leur rapport, V. Šilova et O. Krutov émettent aussi un avis incontestablement favorable, voire admiratif à l'égard de l'organisation du travail chez les « amateurs ». Retenons l'exemple de l'inspection de deux studios : celui du film publicitaire du comité exécutif de Moscou et celui du studio EstReklamFil'm de Tallinn. Dans chacun de ces cas, les experts vérifient les comptes, les contrats et les documents financiers, visionnent les films, lisent leurs scénarios. À cette occasion, à l'encontre du principe de complémentarité dans la filière classique, ils mettent en valeur la spécialisation pratiquée dans les studios institutionnels dans un type de cinéma donné. De leur point de vue, seule la spécialisation favorise la stabilisation des relations avec les commanditaires locaux et, par conséquent, de la thématique traitée par le studio. Elle aboutit aussi à une codification du travail des rédacteurs qui doivent suivre les films du début à la fin, ce qui ne se pratique pas dans la filière classique. Sur le plan logistique et technique, la spécialisation permet d'utiliser plusieurs fois les mêmes décors et, ainsi, de réduire les dépenses. Il en va de même pour les techniques de l'animation qui se standardisent. Les studios institutionnels pratiquent aussi un partage différent des tâches. Ainsi, au sein du studio institutionnel d'Estonie, un rédacteur s'occupe exclusivement des rapports avec les commanditaires, tandis qu'un autre pilote la diffusion des films publicitaires dans les salles et à la télévision. La diffusion est organisée en fonction de consignes données par les commanditaires pour définir la fréquence, les jours et les plages horaires. Les studios institutionnels ont ainsi une longueur d'avance sur leurs homologues classiques, qui n'ont aucune prise sur la diffusion de leurs films.

De la même façon, les experts pointent un autre aspect, de leur point de vue, positif : la réduction du nombre d'employés, qui sont en revanche polyvalents ${ }^{115}$. Ils sont tout aussi admiratifs de l'absence de salariés permanents et citent Tallinn où ce fonctionnement devient régulier : outre les administratifs, un seul opérateur est engagé sur contrat durable. Les autres sont recrutés au coup par coup parmi les employés de la télévision et du studio professionnel Tallinnfil'm. D'ailleurs, face à l'augmentation du nombre de commandes, la direction projette d'embaucher sur un poste pérenne un réalisateur et un opérateur, à qui elle compte imposer des

113. Ibid., 1. 87-88.

114. Ibid., 1. 43.

115. Ibid., 1. 64-66. 
règles de recrutement assez dures. Les professionnels de la TV et du cinéma avec lesquels elle collabore sont confirmés et renommés. Leur salaire est versé seulement pour les périodes de tournage et comprend une prime à l'acceptation du film par le commanditaire ${ }^{116}$. Un élément intrinsèquement lié, le fameux « mécanisme d'intéressement matériel ", préoccupe fortement les experts. Ainsi, le studio du comité exécutif de Moscou pratique le versement d'une prime conditionnée à l'investissement de chaque travailleur à titre individuel (dans les studios classiques, le système prévoit le versement de primes collectives). Pour les experts qui évaluent cette organisation non professionnelle, le studio classique de la basse Volga expérimente aussi ce fonctionnement et cet essai « confirme l'efficacité du travail dans ces nouvelles conditions de la planification et de la stimulation financière ${ }^{117}$. Le débat économique ambiant conditionne cette perception positive que les professionnels ont de ceux qui pourraient être leurs concurrents. À l'évidence, les experts cités adhèrent aux positions d'un groupe de cinéastes qui présentent la stabilité de contrats dans l'industrie cinématographique comme un fléau, entraînant " l'assistanat des créateurs » et un manque d'intérêt pour le résultat de leur travail ${ }^{118}$. Contrairement au Comité de contrôle et aux Comités du cinéma, les personnels des studios impliqués dans les expertises suggèrent que les studios institutionnels ont ainsi supprimé de nombreuses difficultés de la filière classique.

Les initiatives qui naissent par le bas dans la sphère non professionnelle confèrent à ces arguments une fraîcheur et une valeur de preuve pour la filière classique elle-même. Certains dirigeants du cinéma reconnaissent les dysfonctionnements du système qu'ils pilotent. Ainsi, au cours des audits, Vladimir Golovnja, responsable du département du film de non-fiction au sein du comité du Cinéma, écrit aux directeurs des studios classiques du film scientifique de Kiev et de Moscou ainsi qu'aux studios des films de vulgarisation scientifique et documentaire de Géorgie, d'Ouzbékistan et d'Arménie, mettant le Comité de contrôle populaire en copie. Pour résoudre le problème amplement mentionné de la cherté et des délais de fabrication des publicités, il appelle sommairement à « réduire le nombre des lieux, des décors à filmer et à augmenter le rendement des équipes de tournage et des ateliers techniques qui les desservent $\gg{ }^{119}$. Autrement dit, il riposte par la vieille recette de l'économie d'échelle. La vacuité de ces recommandations managériales reste dans le périmètre de l'évaluation classique de la réalisation du

116. Ibid., 1. 68-70.

117. Ibid., 1. 32.

118. J'ai consacré un article à ces deux éléments, portés tels des fanions de la lutte contre « la lourdeur institutionnelle » dans l'économie du cinéma soviétique. Irina Tcherneva, " Rynok protiv plana ? Ėksperimenty v organisacii i oplate truda v sovetskom kino (1961-1976) [Économie de marché contre économie planifiée ? Expérimentations dans l'organisation du travail dans le cinéma soviétique (1965-1976)] Perspectivia.net, 2016. http://www.perspectivia. net/publikationen/shdp/cherneva_rynok et http://www.perspectivia.net/publikationen/shdp/ cherneva_rynok_ii

119. GARF, f. 9527 , op. 1, d. 3034, 1. 78-79. 
plan par les studios. De même, le Comité de contrôle populaire se recroqueville en cette année 1970 sur des sentences ressassées : éviter les doublons dans les sujets, par exemple. Mais cet appel est vain, car le secteur professionnel n'a pas la maîtrise des studios institutionnels. Enfin, le Contrôle reproche à ces derniers de ne pas avoir « de personnel stable, qualifié et salarié », tandis que les cinéastes experts chantent à l'unisson les louanges du recrutement sur contrat ${ }^{120}$. Le pas est cependant franchi : l'absurdité de la réprobation de l'absence de personnel salarié adressée aux studios institutionnels atteste que ces derniers ne sont plus considérés comme non professionnels. Le président du Comité du cinéma Vladimir Baskakov cherche à rendre invisible l'économie hors de son monopole par l'interdiction formelle de quelques initiatives et, à cet égard, suit la décision du Conseil des ministres ${ }^{121}$. En même temps, il décide d'emprunter au cinéma amateur certains modes de fonctionnement pour redynamiser la filière qu'il dirige ${ }^{122}$. Ce faisant, il compte occuper un nouveau terrain, celui du film publicitaire, identifié et investi par les studios institutionnels.

\section{Conclusion}

La structuration du cinéma amateur auprès des usines et des établissements soviétiques est révélatrice d'un déplacement du curseur de la pratique citoyenne vers des impératifs institutionnels. Les laboratoires de photographie et de cinématographie et les studios creés auprès des établissements, des usines, voire auprès des sovnarkhozes, promeuvent les intérêts de ces institutions tutélaires. Leurs films recoupent les intérêts des sphères scientifiques, industrielles, cinématographiques et politiques. Les studios se positionnent comme des hérauts des acteurs locaux et l'intransigeance des réactions du Comité de contrôle d'État et de Parti atteste la difficulté d'accepter la décentralisation entamée sous Hruščev, ainsi que la mise en péril du monopole des institutions d'État et leur maîtrise des prix.

Dans le domaine du cinéma, les studios institutionnels lancent deux défis principaux. D'une part, en se constituant en nouveaux fabricants de films ils forgent une forme de concurrence et posent un défi financier. Certains n'hésitent pas à réclamer la révision du monopole de la filière étatique sur la création des films. De l'autre, ils se positionnent clairement dans le champ du cinéma de commande. Bien que ce secteur soit convoité par la filière traditionnelle, il ne semble pas suffisamment développé au sein de celle-ci et le fonctionnement des studios institutionnels révèle une pénurie de ces films dans l'industrie filmique. Un tel constat fait a posteriori est traditionnel pour l'économie soviétique. Cependant, il ne s'agit pas d'une pénurie

120. Ibid., 1. 85.

121. Ibid., 1. 87-89.

122. Résolution du 18 octobre 1970. GARF, f. 9527, op. 1, d. 3034, 1. 74. 
qui frapperait les consommateurs à titre individuel ${ }^{123}$, elle frappe les ministères. Dès lors apparaissent une formidable malléabilité et une adaptabilité des structures qui se situent en dehors du plan. Une demande et une offre surgissent hors du périmètre traditionnel de l'économie du cinéma. De nombreux aspects de cette nouvelle sphère du cinéma « hors studios professionnels » sont donc susceptibles de ne pas suivre les visées étatiques dans différents champs d'action : activités économiques non encadrées, voire illicites, promotion de méthodes de travail non avalisées par les instances de supervision. À cet égard, le Comité du contrôle est particulièrement vigilant : si la disparité des propositions techniques et scientifiques avancées dans les films institutionnels peut être réduite au coup par coup, il s'avère bien plus délicat d'accepter la métamorphose des studios institutionnels en unités économiques autonomes et autofinancées.

Leur fonctionnement s'enracine dans un mouvement décentralisateur, mais fait aussi écho aux réformes de l'industrie filmique d'État. Les studios institutionnels concurrencent les studios classiques sur le terrain de l'organisation du travail et de la diffusion de leurs produits, de la rapidité de réalisation et de la rémunération des employés. En même temps, ils perturbent les structures professionnelles elles-mêmes, car les praticiens de l'image des studios classiques et les ressources traversent sans cesse les frontières entre les établissements. Les rapports économiques qualifiés au départ d'opaques sont à terme reconnus, aussi bien formellement que dans la communauté professionnelle. L'administration du cinéma et le Contrôle populaire cherchent à encadrer cette compétition, mais leurs réactions viennent constamment après coup. Les audits et les résolutions qui en découlent visent à endiguer le mouvement à l'échelle pansoviétique, en dépit du soutien apporté aux pratiques économiques alternatives par les commanditaires ministériels et par les organisations locales du parti. Ces appuis locaux n'hésitent pas à contester ouvertement le centralisme et, ce faisant, interviennent dans la régulation de la politique économique du cinéma.

En réalité, les transactions marchandes hors plan traversent de part en part la sphère filmique. La reconnaissance ponctuelle de cette activité économique alternative par l'administration du film s'observe tant dans la production que dans la diffusion. Périodiquement (dans les années 1920-1930, 1950-1970), l'administration du cinéma cherche à imposer des types peu rentables de cinéma aux salles pilotées par la Centrale syndicale pansoviétique et par les ministères. Films éducatifs, scientifiques et culturels doivent prioritairement être diffusés par ces circuits qui sortent de l'économie planifiée du cinéma. On peut y voir une ressemblance certaine avec la tentative du Comité de contrôle de ne permettre aux studios institutionnels que de produire du cinéma scientifique et éducatif. Mais aussi, entre les années 1940-1970, les salles syndicales et ministérielles sont tantôt soumises au système centralisé du cinéma, tantôt autonomes. L'administration du film ne parvient pas à contrôler les activités économiques qui se déploient dans ces salles et les Maisons de la culture, qui entrent en concurrence avec le circuit classique 
de diffusion. L'attitude de l'administration du cinéma envers les pratiques dans le réseau non professionnel oscille, là aussi, entre une tendance répressive et une alimentation ponctuelle du système planifié par ces dynamiques alternatives. Ainsi, la filière cinématographique tolère les activités marchandes par le bas et se revigore en procédant à des ajustements professionnels à l'intérieur de structures en apparence immuables.

CERCEC, EHESS, Paris

Fondation pour la Mémoire de la Shoah, Paris

irina.tcherneva@ehess.fr 
